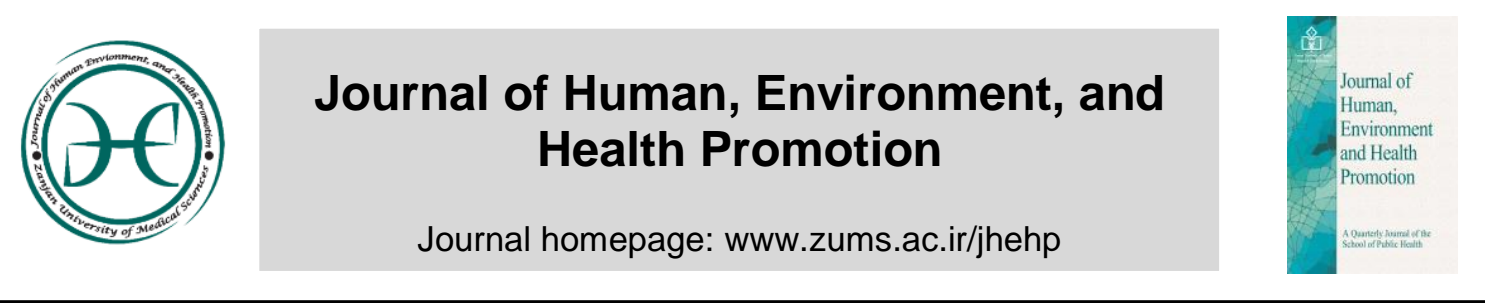

\title{
Tendency to Pregnancy in Women and Their Husbands Attending Health Centers in Zanjan in 2012
}

\author{
Hossein Masoumi-jahandizi a,", Koorosh Kamali a, Ali Mohammadi a \\ ${ }^{a}$ Department of Public Health, School of Public Health, Zanjan University of Medical Sciences, \\ Zanjan, Iran.
}

*Corresponding author.E-mail address: hmjfh@zums.ac.ir

\section{A R T I C L E I N F O \\ Article history: \\ Received September 22, 2015 \\ Accepted November 30, 2015 \\ Article Type: \\ Original Article \\ DOI: $10.29252 /$ jhehp.1.2.105}

Keywords:

Desire for Pregnancy

Spouse

\begin{abstract}
A B S T R A C T
Background: Reproduction is an important population concept throughout the world, and demographists consider it among four main life events. Given Iran's population growth policy, this study was conducted to assess the tendency to pregnancy in women in Zanjan.

Method: From among health centers in five geographical districts in Zanjan (North, South, East, West, and Center), one was randomly selected from each district, and the study was conducted on a total of 400 people. Data were analyzed in SPSS-16 using descriptive and inferential tests including Chi-square, t-test (mean test) and variance analysis.

Results: A total of 186 women (46.5\%) desired pregnancy after marriage and 212 (53\%) did not. Also, 209 husbands (52.2\%) agreed with childbearing after marriage, and 186 (45.5\%) disagreed.

Conclusion: Childbearing can be encouraged among husbands agreeing with pregnancy of their spouse (more than half) and women attending pregnancy counseling and family planning centers.
\end{abstract}

\section{Introduction}

Reproduction is an important population concept, and has always been of interest to various cultures and religions, such that demographists regard it among the four vital events in life.

In Islam, Judaism, and Zoroastrianism, childbearing is held in high esteem.
Accordingly, as followers of one of these religions, Iranians have had and still have many customs in this respect.

In recent years, childbearing and population growth have had many changes. Reproduction is an important demographic concept, and considered among the four vital events by demographers, and since it directly

To cite: Masoumi-jahandizi H, Kamali K, Mohammadi A. Tendency to Pregnancy in Women and Their Husbands Attending Health Centers in Zanjan in 2012. J Hum Environ Health Promot. 2016; 1(2): 1059. 
Influences population growth or decline, it is considered the most important population time-movement factor.

Currently, in Iran, population age pyramid is in transition from young to old. Thus, if 1989 is taken as the starting year for major and consistent decline in pregnancy, Iran's population will be totally composed of old people by 2046 [1].

Total fertility rate determines the number of children replacing their parents. According to UN statistics, fertility rate in Iran was less than 1.8 in 2010. To avoid negative population growth rate in a society, replacement rate should be at least 2.1. The World Bank anticipates that Iran's population growth rate will reach below one percent by 2025 , and Iran will witness a population decline of $0.99 \%$, which implies gradual population decline and extinction of generation [1]. Statistics and decreasing population growth rate in recent years suggest declining population in Iran. Is this true, and if so, what is the reason.

Despite annual addition of one million people to the population of Iran, population is still said to be declining. The reason for this is the declining population growth rate. In Iran, developments in population increase or decrease happened proportional to the whole world and followed the same path as most other countries. With advances in health and death control in various countries, population showed an ascending trend. For instance, in the past, in families with several children, many died at birth or before adulthood. However, following technological advances affecting improvements in health and lifestyle throughout the world, the majority of children born survived, which led to population increase in most countries. Similarly, Iran also witnessed population growth at this stage and entered population explosion stage as in most countries [2].

Following population explosion of 1981-89, most young population of marrying age have not married yet, and this proverbially "not settled down" people, especially young girls, have turned into a serious trouble [2].

A major contributing factor to the decline in childbearing is older marrying age, which is affected by other factors such as individualism, rulerchild, and .... . Today, mean marrying age has increased due to the strengthened nuclear family and financial problems in industrial societies [3]. Individualism per se can be a consequence of development of nuclear family, and can end in reduced accountability, older marrying age, and increased divorce rate with subsequent reduced child bearing [4].

The bitter experience of parents' divorce wards off young people from marrying, which results in population decline. Divorce has profound socioeconomic, psychological, legal, and parental consequences for divorced men and women, their children, and the society, and irrespective of the cause, leads to reduced childbearing in the family [1]. Financial problems are also highly decisive in reduced fertility and the number of children in a family [1].

Women's higher education level has led to their greater desire for participation in the job market, which has increased their employment rate compared to the past. Women's higher involvement outside home has reduced their willingness to bear children, and has adversely affected childbearing rate in Iran [1].

Following industrialization of the West, nuclear family appears to be the ruling form of a family in both industrial and other parts of the world. Similarly, currently in Iran, nuclear family is the leading form of family [1]. 
Urbanization has led to people's higher expectations for better life, has burdened them with increased cost of living, which has changed women's reproductive behaviors in favor of smaller households and fewer children. Spread of urbanization leads to further development of nuclear family, which in turn adversely affects number of children [5]; higher expectations of children, increased parental care and attention toward children, increased parental expenditure on children, [6].

In such circumstances, childbearing rate naturally reduces; since the ability to meet children's expectations and excessive parental attention are huge impediments to having more children [6].

In recent years, women's desire to work and earn has increased, which has led to increased women's employment and availability in the job market. As a consequence, there has been a change in attitudes toward marriage, maternal and wife roles and domestic work, which has been followed by a kind of role change. This attitude change has propagated down from feministic beliefs in western societies to other countries, including Iran [1].

Development of the consumer culture naturally increases costs of living, and is not conducive to having more children; hence reduced number of children [7].

American researchers have shown that the best childbearing age for women is between 20 and 30 years, but there is no real need to wait until then. Financial stability and physical and mental health are the best criteria for individuals to decide to extend their family. Studies have shown that reproductive power declines with aging, and large birth spacing increases likelihood of miscarriage. However, couples should know that pregnancy does not necessarily occur immediately with sexual relationship without contraception, and it may even take a year for it to happen and even then, pregnancy takes nine months, which gives parents sufficient time to plan for parenthood [7].

Women are the most fertile at the age of 25, when chances of pregnancy in the monthly cycle are $25 \%$, which reduces to $6 \%$ at the age of 40 . Studies conducted in Brazil have shown that men's fertile powers diminish after the age of 41 due to reducing quality of sperm with aging [8].

This study was conducted given demographists' concerns about declining population and national policies in favor of population growth, and also absence of research in Zanjan.

\section{Materials and Methods}

This study was conducted in the city of Zanjan in 2012. From among health centers in five geographical districts in Zanjan (North, South, East, West, and Center), one was randomly selected from each district, and study was conducted on a total of 400 people. Data were collected using a researchermade questionnaire, containing 15 demographic questions and 25 about women's knowledge of two contraception methods (women's tubal ligation, and use of contraceptive pills) and their and their husbands' views on pregnancy. Reliability of questionnaire was confirmed with Cronbach's alpha $(\alpha=90 \%)$. Data were analyzed in SPSS16 using descriptive and inferential tests including Chi-square, t-test (mean test) and variance analysis. Each correct answer scored 1 mark, and wrong or no comment answers scored zero.

\section{Results}

In this study, the youngest participant was 16 years old and the oldest 53, and mean age was 33.72 years. The shortest duration of marriage 
was one year, and the longest 37 years, and mean duration 11.91 years.

Of participating women, 359 $(89.8 \%)$ were housewives, $39(9.8 \%)$ were employed, and $2(0.5 \%)$ were selfemployed, 228 participants $(57 \%)$ were born in the city and $167(41.8 \%)$ in rural areas, 21 participants $(5.2 \%)$ were illiterate, $199(49.8 \%)$ were able to read and write, 130 (32.5\%) had diploma, 45 $(11.2 \%)$ university degree, and 10 $(1.2 \%)$ degree and higher. Of participants' husbands, $2(0.5 \%)$ were unemployed, 105 (26.2\%) employed, and $291 \quad(72.8 \%)$ self-employed. Furthermore, $17.2 \%$ (82 women) had learnt About contraceptive methods from family, $66 \%$ (315 women) from health centers, $5 \%$ (20 women) the radio and $\mathrm{TV}, 3.2 \%$ (11 women) papers, $6.1 \%$ (29 women) friends, and 4.2\% (20 women) other. 209 husbands (52.2\%) agreed with having children immediately after marriage and 186 (46.5\%) disagreed. 167 husbands (42\%) agreed with use of contraception by themselves and 223 (55.8\%) disagreed.

Table 1: Frequency distribution of participants' and their husbands' desire for pregnancy.

\begin{tabular}{|c|c|c|c|c|c|c|}
\hline & \multicolumn{2}{|c|}{ Desire for pregnancy } & \multicolumn{2}{|c|}{ No desire for pregnancy } & \multicolumn{2}{|l|}{ Total } \\
\hline & Number & $\%$ & Number & $\%$ & Number & $\%$ \\
\hline Participants & 186 & $46.5 \%$ & 212 & $53 \%$ & 398 & $99.5 \%$ \\
\hline $\begin{array}{l}\text { Participants' } \\
\text { husbands }\end{array}$ & 209 & $52.2 \%$ & 186 & $46.5 \%$ & 395 & $98.8 \%$ \\
\hline
\end{tabular}

\section{Discussion and Conclusion}

In this study, $17.2 \%$ (82 women) had learnt about contraceptive methods through family, $66 \%$ (315 women) through health centers, 5\% (20 women) the radio and $\mathrm{TV}, 3.2 \%$ (8 women) papers, $6.1 \%$ (29 women) friends, and $4.2 \%$ (20 women) other. In a similar study conducted in India, $54.4 \%$ of participants had learnt about contraceptives through radio and TV, $37.7 \%$ through family and friends, and $7.9 \%$ through healthcare providers. A study showed that family and friends are the most important sources of such information with $67.3 \%$, followed by TV $32 \%$, doctors $21 \%$, papers $10.7 \%$, and school 6\% [9]. Accordingly, to encourage pregnancy, we can resort to TV and health centers because they are the most important sources for women.

Husbands of participating women agreed with having children immediately after marriage (52.2\%) 209 and $186(46.5 \%)$ disagreed. In a study conducted in Zanjan, $73.2 \%$ had no desire for pregnancy immediately after marriage, unlike the rest $(26.8 \%)$ that wanted children immediately after marriage. Men had significantly greater desire for having children than women $(P=0.007)$ [8]. Hence, one of the ways

to encourage women is through motivation of their husbands. In a family, husband and wife usually decide about having children, and husband's consent can have an important role.

Husbands that agreed with use of contraception by themselves (42\%) 167 and $223(55.8 \%)$ disagreed.

About the most important source of education about contraception, $10.4 \%$ (44) reported family, $76.4 \%$ (325) reported health center, $5.2 \%$ (22) Radio and TV, 2.3\% (10) papers, $1.9 \%$ [8] friends, and $3.8 \%$ (16) others. In their study, Peterson \& Rosenberg emphasized the huge role of family planning officials in improving contraception methods [10]. According to the present study and Peterson \& Rosenberg study, health centers can 
have a huge role in teaching fertility issues, and can provide appropriate education to encourage pregnancy.

In this study, 186 women $(46.5 \%)$ agreed with pregnancy immediately after marriage, but $212 \quad(53 \%)$ disagreed. In another study, $3.53 \%$ of women showed no desire for having another child and $7.46 \%$ wanted one or more children in the future [10], which concur with the present study.

The high percentage of husbands agreeing with pregnancy of their wives after marriage, and also women's reference point for education about pregnancy and contraception can be used to encourage women's pregnancy.

\section{Acknowledgement}

We thank University Research Deputy, Research Director, health center experts, especially Ms ZamaniBargesteh She, and all those that helped in this study.

\section{References}

1. WWW.Shafaonline.ir/Site.

2. Farhangi, A. Kavoosi, E. Cultures and Organizations; Planning Mind. Tehran, Publications Res Institute for Strateg Stud. 2008: 114.

3. Khalagabadi, Farahani F. Reasons for Low Fertility Rate among Iranian Women. Iran Population Newsletter. 2011: 37 .

4. Population and Housing Census, 1966 to 2011. http:// Wikipedia.org.

5. Mahdavi MS. Ghanimati H. The Ruler Child. Humanitarian Sci J. 2002; 35: 114.

6. Eivazi, GH. TV and Consumer Lifestyle, Periodical of Women and Family Defense Culture. 2009; 16:155.

7. Preachy R, Shava C, Ankar DC, Khati Benita J. A Study of Knowledge
Attitude and Practice of Family Planning among the Women of Reproductive age in Sikkim. J Obstet Gynecol India. 2008; 58(1): 63 - 67.

8. Reena S, Dhirendra Kumar S, Radha J, Sharma Neela K, Sushmita S. Contraceptive Knowledge Attitude and Practice Survey. J Obstet Gynecol India. 2005; 55(6): 546-50.

9. Kazemi, A, Torabi Z, Kosha A, Kosha M. Evaluation of Knowledge of the People Intending Marriage about Contraception Methods in Zanjan City. Sci J Zanjan Univ Med Sci. 2001; 34: 26-32.

10. Peterson LS, Oakley D, Potter LS, Darroch JE. Women's Efforts to Prevent Pregnancy: Consistency of Oral Contraceptive Use. Fam Plan Persp. 1998; 1: 19-23. 